\title{
Применение метода твердофазного ионного обмена для насыщения наноионитов лекарственными средствами в отсутствие фонового электролита
}

\author{
(C) 2020 Колотилина Н.К., Шайхина С.У. \\ Институт геохимии и аналитической химии им. В.И. Вернадского РАН, Москва \\ Поступила в редакцию 19.11.2020 г.
}

DOI: $10.17308 /$ sorpchrom.2020.20/3136

В настоящее время актуальной является проблема разработки систем целевой доставки лекарственных средств с использованием наноразмерных носителей. В этой связи большой интерес проявляется к полимерным материалам в силу их физических свойств, большой емкости по переносу лекарств, биосовместимости и низкой токсичности. Наноразмерные иониты (НИО), полученные в виде устойчивых гидрозолей (катионных - НИК и анионных - НИА) по своим характеристикам имеют потенциал для использования в качестве доставщиков ионных форм лекарственных средств

Целью данной работы является описание способа получения чистых лекарственных форм на базе наноионитов методом твердофазного ионного обмена, который возможен только в случае, если одной фазой является макроионит, а другой - наноионит той же полярности.

Способ проиллюстрирован на примере получения НИА в форме аниона ди-N-метилглюкаминовой соли гадопентата (торговое название «Магневист») - контрастного агента при МРТ диагностике. Получение препарата целевой доставки актуально из-за потенциальной токсичности соединений гадолиния.

Было проведено независимое изучение твердофазного ионного обмена для систем с макроионитами. Эксперимент заключался в переносе определенного иона с одной анионообменной колонки (1) в (Br-) форме на другую, такую же, но в ОН-форме (2), методом пропускания наногидрозоля НИА известной концентрации через систему последовательно соединенных колонок. Контроль степени переноса бромида осуществлялся путем измерения концентрации $\mathrm{Br}$, смываемого с колонки 2 раствором натриевой щелочи после разъединения колонок. Бромид определяли хроматографически. Сведя баланс количества эквивалентов пропущенного НИА и смытого бромида, установили, что твердофазный ионный обмен прошел в полном объеме. Бромид полностью перенесен с колонки 1 на колонку 2.

Получив доказательство эффективности способа твердофазного ионного обмена, применили этот подход для получения частиц наногидрозоля НИА, нагруженных анионом [Gd-ДТПА] ${ }^{2-}$. Использовали макроанионит и НИА в солевых формах, так как комплекс гадолиния устойчив в нейтральных и слабокислых средах. Контроль полноты перевода $\mathrm{AB}-17\left(\mathrm{Cl}^{-}\right)$в форму $[\mathrm{Gd}-Д Т П А]^{2-}$ проводили методом одновременного ионохроматографического определения обоих анионов в пробах, отбираемых на выходе из колонки. Далее переносили комплекс гадолиния в наногидрозоль ( $\left.\mathrm{Br}^{-}\right)$, пропуская НИА через колонку с макроанионитом в форме [Gd-ДТПА] ${ }^{2-}$. Таким образом, в процессе твердофазного ионного обмена насытили гидрозоль НИА анионом [Gd-ДТПА $]^{2-}$, вытесняя бромид в фазу макро анионита. Полученный методом твердофазного ионного обмена гидрозоль в форме [Gd-ДТПА] $]^{2-}$ передан на испытание в РНИМУ им. Н.И. Пирогова.

Благодаря возможностям твердофазного ионного обмена можно легко и быстро переводить наноиониты в ионную форму лекарственного средства, насыщать полистирольные наночастицы, не теряя при этом дорогостоящие компоненты в окружающем растворе. Отсутствие посторонних химических примесей в полученном препарате имеет первостепенное значение при его медицинском применении, так как при этом сводятся к минимуму нежелательные побочные эффекты.

Ключевые слова: твердофазный ионный обмен, наноиониты, наноионообменник, гидрозоль, наночастицы, направленная доставка лекарственных средств. 


\section{Введение}

В настоящее время в медицине и фармакологии все большую актуальность приобретает метод направленного транспорта лекарственных средств, позволяющий увеличить концентрацию лекарств в определенной локальной области организма и блокировать или сильно ограничить их накопление в здоровых органах и тканях. Ocoбенно актуальна целевая доставка лекарств при онкологии, где традиционная химиотерапия вызывает интоксикацию всего организма пациента [1]. Направленный транспорт позволяет повысить продолжительность и эффективность действия лекарства за счет увеличения его локальной концентрации в целевом органе при снижении нежелательных побочных эффектов. В последние десятилетия особенно возрос интерес к полимерным наночастицам как средствам доставки $[2,3]$. Преимущества полимерных частиц - их стабильность, низкая токсичность, возможности эффективного включения лекарственных веществ различной физико-химической природы и фармакологических свойств в полимерную матрицу. Нагруженные лекарством полимерные наночастицы способны проникать из кровеносной системы в воспаленные ткани и опухоли и накапливаться в них благодаря рыхлости и негерметичности сосудистой системы больных тканей [4].

Наноразмерные иониты (НИО) (рис.1), полученные в виде устойчивых гидрозолей (катионных - НИК и анионных - НИА) [5-8] по своим свойствам имеют потенциал для использования как в аналитической химии $[5,8]$, так и в качестве доставщиков ионных форм лекарственных средств $[6,7]$. Обладая большой ионообменной емкостью, частицы НИО способны нести высокую нагрузку ионных форм лекарственных средств. Особую ценность имеют чистые лекарственные формы - гидрозоли, не содержащие никаких других компонентов, кроме наночастиц с требуемой ионной формой. Это важно для предотвращения нежелательных побочных воздействий посторонних примесей в гидрозоле на организм.

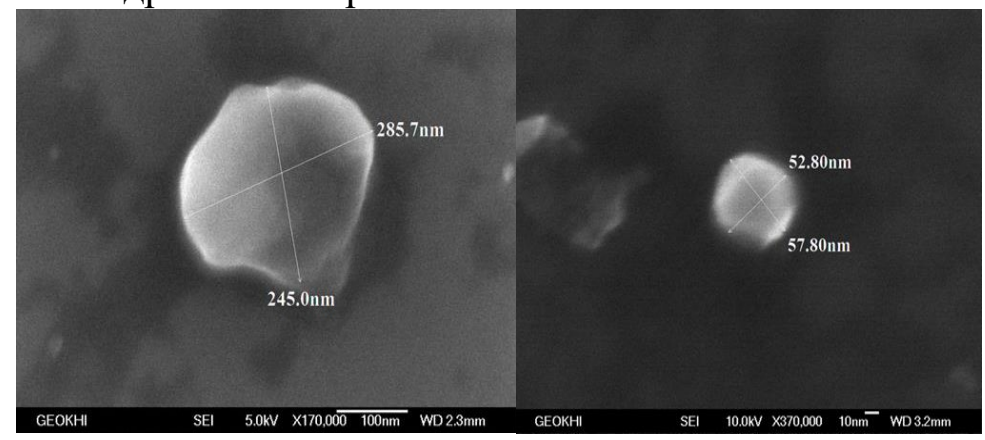

a

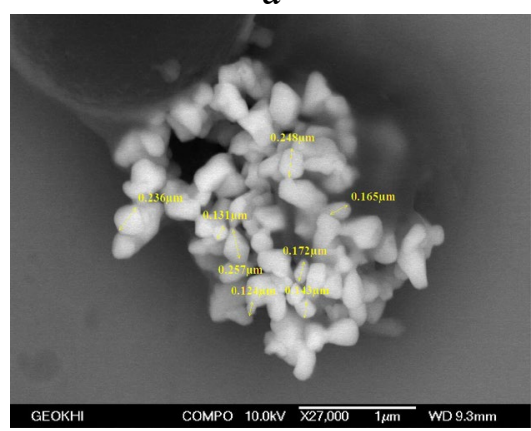

6

Рис. 1. Электронные микрофотографии частиц наноионитов: а) нанокатионит (НИК), б) наноанионит (НИА). Дипазон размеров наночастиц от 50 до 250 нм

Fig.1. Electron micrographs of nanoionite particles: a) nanocationite (NIC), b) nanoanionite (NIA). Nanoparticle size range from 50 to $250 \mathrm{~nm}$ 
Целью данной работы является описание способа получения чистых лекарственных форм на базе наноионитов методом твердофазного ионного обмена, который возможен только в случае, если одной фазой является макроионит, а другой наноионит той же полярности.

Способ проиллюстрирован на примере получения НИА в форме аниона ди-Nметилглюкаминовой соли гадопентата (торговое название - «Магневист»). Магневист применяется в медицине при диагностике как контрастное вещество для визуализации патологий различных органов методом магнитно-резонансной томографии. В последние годы активно обсуждается проблема потенциальной токсичности препаратов гадолиния [9-11], поэтому особенно важно минимизировать его попадания в здоровые органы пациентов, локализовав в больном органе методом нацеленной доставки целесообразного количества.

\section{Экспериментальная часть}

При проведении экспериментов использовали следующие реагенты: AB-17 товарный анионит зернением 0.3-0.5 мм, концентрированную соляную кислоту $\mathrm{HCl}$; натриевую щелочь $\mathrm{NaOH}$; карбонат натрия $\mathrm{NaHCO}$ и бикарбонат натрия $\mathrm{Na}_{2} \mathrm{CO}_{3}$; роданид аммония $\mathrm{NH}_{4} \mathrm{SCN}$; бромид натрия $\mathrm{NaBr}$, товарный лекарственный препарат магневист. Согласно описанию флакон с препаратом содержал $20 \mathrm{~cm}^{3}$ водного раствора ди-N-метилглюкаминовой соли гадопентата с концентрацией последнего 469.01 г/дм ${ }^{3}\left(0.5 \mathrm{M}\right.$ раствор соединения $\left.\mathrm{C}_{28} \mathrm{H}_{54} \mathrm{GdN}_{5} \mathrm{O}_{20}\right)$. Концентрация аниона [Gd-ДТПА] ${ }^{2-}$ (где ДТПА - диэтилентриаминпентауксусная кислота) составляла, таким образом, 0.5 М. Содержимое флакона разбавляли в 25 раз для получения 0.02 М раствора магневиста. Все необходимые растворы и суспензии готовили, используя бидистиллированную воду и реактивы квалификации не ниже «Х.Ч.».

В работе использовали ионный хроматограф «Цвет 3006» (Дзержинск, РФ); роторный дезинтегратор; шаровую мельницу; центрифугу; перистальтический насос. Контроль размера наночастиц осуществляли с помощью электронного микроскопа «Tescan Mira» (Чехия).

Основные аналитические определения проводили методом ионной хроматографии. Элементы хроматографической системы: насос с максимально допустимым давлением 8 МПА; разделяющая колонка 5 (внутр. диам.)х120 мм с сорбентом КанКАст зернением - 14 мкм, емкостью - 0.0102 мг-экв $/ \mathrm{cm}^{3}$; подавительная колонка: 6х200 мм, с катионитом CПC SAC (8\%) зернением 15 мкм, емкостью - 2 мг-экв/ $\mathrm{cm}^{3}$; дозирующая петля объемом 30 мкдм $^{3}$, кондуктометрический детектор. Состав элюента: $3.6 \mathrm{mM} \mathrm{NaHCO} 3 ; 3$ мM Na $2 \mathrm{CO}_{3} ; 0.03$ мM NH $4 \mathrm{SCN}$. Скорость потока элюента $-2 \mathrm{~cm}^{3} /$ мин. Относительная ошибка определения хлорида составляла $2.5 \%$, бромида $3.2 \%$, аниона Gd-ДТПА $]^{2}-5 \%$.

Получение наноразмерных ионитов проводили в несколько стадий [6]:

1 - подготовка 2 дм $^{3}$ исходного товарного анионита AB-17 (0.3-0.5 мм) - перевод в нужную ионную форму $\left(\mathrm{OH}^{-}\right)$, отмывка и сушка;

2 - двухступенчатый размол - до десятков мкм с помощью роторного дезинтегратора, и до требуемой дисперсности (0.05-0.3 мкм) в ходе дальнейшего размола с применением шаровой мельницы;

3 - получение водной суспензии продукта размола и несколько стадий седиментации;

4 - выделение гидрозоля НИО путем отсечения центрифугата от осадка, полученного в условиях длительного интенсивного центрифугирования водной суспензии последней стадии седиментации (1 час при скорости вращения 7000 об/мин). 
5 - выделение гидрозоля НИО путем отсечения центрифугата от осадка, полученного в условиях длительного интенсивного центрифугирования водной суспензии последней стадии седиментации (1 час при скорости вращения 7000 об/мин).

Гидрозоль подвергали дополнительной очистке от примесей, пропуская со скоростью $0.7 \mathrm{~cm}^{3} /$ мин через колонки диаметром 4 мм и объемом $6 \mathrm{~cm}^{3}$ сперва с катионитом СПC SAC $\left(\mathrm{H}^{+}\right)$, затем с анионитом Дауэкс 2x10 (200-400 меш) в $\mathrm{OH}^{-}$форме. Получали НИА в стандартном состоянии - НИА $\left(\mathrm{OH}^{-}\right)$на фоне воды.

Определяли концентрацию НИА $\left(\mathrm{OH}^{-}\right)$до и после очистки методом обратного кислотно-основного титрования соляной кислотой с индикатором фенолфталеином. К точной аликвоте НИА добавляли избыточное количество соляной кислоты, тщательно перемешивали и спустя 2 мин. титровали избыток кислоты щелочью известной концентрации. Концентрацию НИА по функциональным группам рассчитывали по формуле:

$$
\mathrm{C}_{\mathrm{HИА}}=\frac{\mathrm{C}_{\mathrm{HCl}} V_{\mathrm{HCl}}-\mathrm{C}_{\mathrm{NaOH}} V_{\mathrm{NaOH}}}{V_{\text {НИА }}},
$$

где $\mathrm{C}_{\mathrm{HCl}}, \mathrm{V}_{\mathrm{HCl}}$ - концентрация и объем добавленной соляной кислоты, $\mathrm{C}_{\mathrm{NaOH}}, \mathrm{V}_{\mathrm{NaOH}}-$ концентрация и объем щелочи, затраченной на титрование, $\mathrm{V}_{\text {ниА }}$-аликвота анализируемого золя. По результатам титрования $\mathrm{C}_{\text {ниА }}=0.011 \mathrm{M}$. Относительная ошибка обратного титрования не превышала $3 \%$.

\section{Обсуждение результатов}

Реакция твердофазного ионного обмена описывается уравнением:

$$
A x^{+} X^{-}+a x^{+} B^{-} \stackrel{\mathrm{H}_{2} \mathrm{O}}{\longrightarrow} A x^{+} B^{-}+a x^{+} X^{-}
$$

где $A x$ - макроанионит; $a x$ - наноанионит; $X$ и $B$ - противоионы.

Попытки наблюдения и изучения процесса твердофазного ионного обмена делались ранее для систем с макроионитами (зернение 0.5 мм) [12]. Однако применению этого процесса препятствовала очень медленная кинетика. В системах с наноионитами кинетика характеризуется временами в миллионы раз меньшими, что позволяет проводить твердофазный ионный обмен в режиме, обычном для динамических сорбционных экспериментов. В таких системах твердофазный ионный обмен осуществляется между макроионитом, наполняющим колонку, и гидрозолем наноионита той же полярности $[6,7]$.

Таким образом, в процессе твердофазного ионного обмена макроионит передает гидрозолю наноионита необходимый ион, полностью насыщая его ионообменную емкость, что позволяет использовать этот процесс для перевода НИА в любую требуемую форму без попадания в водную фазу каких-либо веществ.

Прежде чем приступить к получению лекарственного образца на основе гидрозоля проводили независимое изучение твердофазного ионного обмена для систем с макроионитами (рис. 2).

Наполняли две одинаковые колонки анионитом АВ-17 (рис. 2). Колонку 1 переводили в форму бромида, пропустив $120 \mathrm{~cm}^{3} 0.5 \mathrm{M}$ раствора $\mathrm{NaBr}$. Бромид был выбран в качестве реперного аниона для удобства хроматографического определения четкое место на хроматограмме без перекрытия другими пиками, заведомое отсутствие его примесей в водных растворах.

Колонку 2 переводили в гидроксильную форму, пропустив $100 \mathrm{~cm}^{3} 0.25 \mathrm{M}$ раствор $\mathrm{NaOH}$, скорость пропускания в обоих случаях $-0.9 \mathrm{~cm}^{3} /$ мин. Колонки тщательно промывали бидистилированной водой $\left(150 \mathrm{~cm}^{3}\right)$, соединяли, определяли свободный объем системы $-4.9 \mathrm{~cm}^{3}$. 
На первом этапе эксперимента через систему колонок со скоростью $0.5 \mathrm{~cm}^{3} /$ мин пропускали $100 \mathrm{~cm}^{3}$ НИА(ОН). Первые $4.9 \mathrm{~cm}^{3}$ отбрасывали, остальное количество собирали в колбу. После этого систему колонок промывали с той же скоростью $100 \mathrm{~cm}^{3}$ бидистилированной воды (первые $4.9 \mathrm{~cm}^{3}$ отмывочного раствора добавляли в емкость с НИА).

1

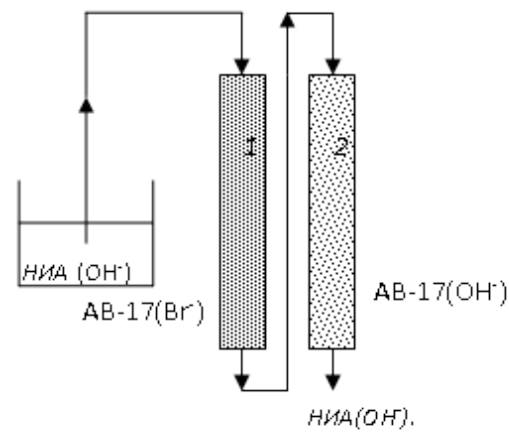

Рис. 2. Установка для изучения процесса твердофазного ионного обмена. Колонки 1 и 2 диаметром 4 мм и объемом загрузки $6 \mathrm{~cm}^{3}$. Зернение анионита AB-17 0.3-0.5 мм. Колонка 1 - AB-17 (Br-), колонка 2 - AB-17( $\left.\mathrm{OH}^{-}\right)$.

Концентрация НИА $(\mathrm{OH})^{-}-0.011 \mathrm{M}$. Скорость пропускания НИА $-0.5 \mathrm{~cm}^{3} / \mathrm{Mин}$. Концентрация $\mathrm{NaOH}-0.2 \mathrm{M}$.

Fig. 2. The unit for studying the process of solid-phase ion exchange. Columns 1 and 2 with a diameter of $4 \mathrm{~mm}$ and a loading volume of $6 \mathrm{~cm}^{3}$. The graining of the AV-17 anionite is $0.3-0.5 \mathrm{~mm}$. Column $1-\mathrm{AB}-17\left(\mathrm{Br}^{-}\right)$, column $2-\mathrm{AB}-17\left(\mathrm{OH}^{-}\right)$. NIAOH) ${ }^{-}$concentration is $0.011 \mathrm{M}$. NIA flowing rate $-0.5 \mathrm{~cm}^{3} / \mathrm{min}$. NaOH concentration $-0.2 \mathrm{M}$.

На втором этапе эксперимента колонку 2 отсоединяли, дополнительно промывали противотоком водой $\left(50 \mathrm{~cm}^{3}\right)$ и пропускали через колонку $0.2 \mathrm{M}$ раствор $\mathrm{NaOH}$ со скоростью $0.46 \mathrm{~cm}^{3} /$ мин, смывая бромид-ион. Выходная кривая бромида представлена на рис. 3. На выходе из колонки отбирали пробы по 50 и $100 \mathrm{~cm}^{3}$. В пробах определяли концентрацию бромида методом ионной хроматографии.

Таблица 1. Результаты определения суммарного количества бромида, смытого с колонки 2.

Table 1. Results of determining the total amount of bromide washed out from the column 2.

\begin{tabular}{|c|c|c|c|c|c|}
\hline № фракции & $\begin{array}{c}\text { Объем фрак- } \\
\text { ции, см }\end{array}$ & $\begin{array}{c}\text { Пропущ. } \\
\text { объем } \\
\text { NaОН, см }\end{array}$ & $\begin{array}{c}\text { Конц. } \mathrm{Br}^{-} \text {в } \\
\text { пробе, } \\
\text { мг/дм }\end{array}$ & $\begin{array}{c}\text { Кол. Вr- в } \\
\text { пробе, мэкв }\end{array}$ & $\begin{array}{c}\text { Сумм. кол. } \mathrm{Br}^{-}, \\
\text {мэкв }\end{array}$ \\
\hline 1 & 50 & 50 & 796.7 & 0.498 & 0.498 \\
\hline 2 & 50 & 100 & 268.0 & 0.168 & 0.666 \\
\hline 3 & 50 & 150 & 147.7 & 0.092 & 0.758 \\
\hline 4 & 50 & 200 & 110.2 & 0.069 & 0.827 \\
\hline 5 & 50 & 250 & 75.5 & 0.047 & 0.874 \\
\hline 6 & 100 & 350 & 34.8 & 0.041 & 0.915 \\
\hline 7 & 100 & 450 & 26.6 & 0.034 & 0.949 \\
\hline 8 & 50 & 500 & 29.4 & 0.018 & 0.967 \\
\hline 9 & 50 & 550 & 41.87 & 0.026 & 0.993 \\
\hline
\end{tabular}


Через систему колонок был пропущен объем гидрозоля, содержащий 1.10 мэкв НИА. Согласно таблице 1 с колонки 2 было смыто щелочью 0.993 мэкв бромида. Из полученных результатов (табл.1) ясно, что бромид практически в эквивалентном количестве перенесен из колонки 1 на колонку 2.

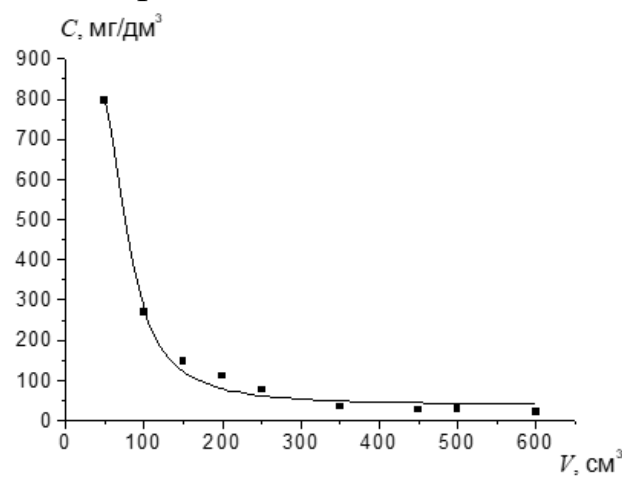

Рис. 3. Выходная кривая бромида при смывании его щелочью с колонки 2, пропускали $0.2 \mathrm{M}$ раствор $\mathrm{NaOH}$ со скоростью $0.46 \mathrm{~cm}^{3} / \mathrm{Mин,} \mathrm{cm}$. рис. 2.

Fig. 3. The elution curve of bromide washed with alkali from the column 2, $\mathrm{NaOH}$ solution was passed with a flow rate of $0.46 \mathrm{~cm}^{3} / \mathrm{min}$, see Fig. 2 .

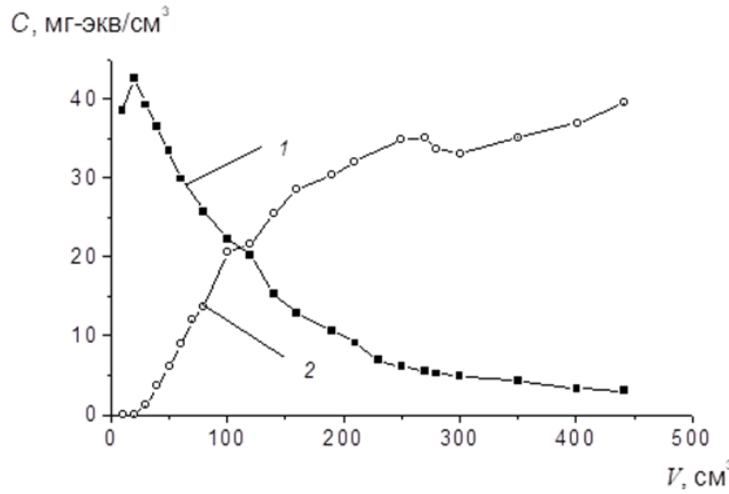

Рис. 4. Выходные кривые вытеснения хлорида (1) и поглощения [Gd-ДТПА $]^{2-},(2)$ при пропускании $0.02 \mathrm{M}$ раствора магневиста через колонку с АB-17( $\left.\mathrm{Cl}^{-}\right)$. Объем колонки $-6 \mathrm{~cm}^{3}$, скорость потока $0.48 \mathrm{~cm}^{3} /$ Мин.

Fig. 4. The elution curve of chloride displacement (1) and absorption of [Gd-DTPA] ${ }^{2-},(2)$ when $0.02 \mathrm{M}$ Magnevist solution was passed through a column with $\mathrm{AB}-17\left(\mathrm{Cl}^{-}\right)$. Column volume $-6 \mathrm{~cm}^{3}$, flow rate $-0.48 \mathrm{~cm} / \mathrm{min}$.

Убедившись в правомерности применения способа твердофазного ионного обмена, приступили к процессу приготовления лекарственного образца на основе гидрозоля.

Прежде всего, готовили макроанионит в форме аниона [Gd-ДТПА] ${ }^{2-}$. Поскольку комплекс гадолиния устойчив в нейтральной и слабокислой среде, «участники» процесса твердофазного обмена - макроанионит и НИА - должны быть переведены в нейтральную солевую форму.

Ионообменную колонку (диаметром 4 мм, объемом $6 \mathrm{~cm}^{3}$ ) с анионитом AB$17\left(\mathrm{OH}^{-}\right)$переводили в хлоридную форму, для чего через колонку пропускали $120 \mathrm{~cm}^{3}$ $0.25 \mathrm{M}$ соляной кислоты со скоростью $0.7 \mathrm{~cm}^{3} /$ мин. Затем колонку отмывали $100 \mathrm{~cm}^{3}$ бидистиллированной воды.

Ионообменную колонку с AB-17 $\left(\mathrm{Cl}^{-}\right)$переводили в форму [Gd-ДТПА] ${ }^{2-}$, пропуская через нее со скоростью $0.48 \mathrm{~cm}^{3} /$ мин $0.02 \mathrm{M}$ раствор магневиста. Для контроля процесса перехода анионита в нужную форму на выходе из колонки отбирали фракции по $10 \mathrm{~cm}^{3}$ и одновременно определяли хлорид и анион [Gd-ДТПА] ${ }^{2-}$ методом ионной хроматографии.

Через колонку было пропущено $441 \mathrm{~cm}^{3} 0.02 \mathrm{M}$ раствора магневиста - 17.6 мэкв. Площадь под кривой 2 на рис. 4 , соответствующая количеству поглощенного вещества, равна 11.8 мэкв. Следовательно, анионитом поглощено 5.8 мэкв [GdДТПА $]^{2-}$, что соответствует емкости колонки с AB-17 - 0.98 мэкв/ $\mathrm{cm}^{3}$. Таким образом, весь анионит перешел в нужную форму.

Солевую форму гидрозоля получали, пропуская $100 \mathrm{~cm}^{3} \mathrm{HИA}\left(\mathrm{OH}^{-}\right)$со скоростью $0.46 \mathrm{~cm}^{3} /$ мин через колонку того же размера с AB-17 в бромидной форме. Гидрозоль НИА( $\left.\mathrm{Br}^{-}\right)$собрали на выходе из колонки. 
Затем $90 \mathrm{~cm}^{3}$ НИА в бромидной форме пропускали со скоростью $0.46 \mathrm{~cm}^{3} /$ мин через колонку с анионитом AB-17 в форме [Gd-ДТПА $]^{2-}$. Фильтрат - гидрозоль, нагруженный магневистом, собрали. Таким образом, в процессе твердофазного ионного обмена перенесли анион [Gd-ДТПА $]^{2-}$ с макроанионита AB-17 в гидрозоль НИА, вытеснив бромид в фазу макроанионита. Полученный методом твердофазного ионного обмена гидрозоль в форме $[\mathrm{Gd}-Д Т П А]^{2-}$, передан на испытание в РНИМУ им. Н.И. Пирогова.

\section{Заключение}

Разработка средств направленной доставки лекарственных веществ, таких как наноиониты (НИА и НИК), является перспективным направлением по созданию лекарственных препаратов нового поколения. Размер частиц наноионитов позволяет им преодолевать физиологические барьеры и доставлять лекарства целенаправленно к больным органам.

Способ твердофазного ионного обмена предоставляет возможность легко и быстро переводить наноиониты в форму лекарственного средства, насыщать полистирольные наночастицы, не теряя при этом дорогостоящие компоненты в окружающем растворе. Полученные в результате лекарственные формы представляют собой твердые полимерные частицы, обладающие всеми необходимыми свойствами. Они способны проникать в больные ткани и накапливаться в них, при этом сохраняя лекарственное вещество в процессе транспортировки и донося его до цели. Отсутствие посторонних химических примесей в полученном препарате имеет первостепенное значение. Химическая чистота препарата сводит к минимуму нежелательные побочные эффекты при его медицинском применении. Наноиониты, обладая большой емкостью и возможностью насыщения ионной формой лекарства, способны существенно увеличить локальную концентрацию действующего вещества в месте назначения, одновременно снижая его токсичность для всего организма.

\section{Список литературы}

1. Perez-Herrero E. // Eu.J Pharm. and Biopharm. 2015. Vol. 93. pp. 52-79. doi: 10.1016/j.ejpb.2015.03.018

2. Duncan R. Vicent M. // Adv. Drug Deliv. Rev. 2013. 65. pp. 60-70. doi: 10.1016/j.addr.2012.08.012

3. Ekladious I., Colson Y, Grinstaff M. // Natur. Rev. Drug Discov. 2019. Vol. 18. pp. 273-294. doi: 10.1038/s41573-018-0005-0

4. Matsumura Y, Maeda H. // Cancer Research.1986. Vol. 46. No 12. pp. 6387-6392.

5. Долгоносов А.М., Колотилина Н.К., Ядыков М.С., Бурмистров А.А. // Журн. аналит. химии. 2013. Т. 68. № 5. С.490-496.

6. Долгоносов А.М., Хамизов Р.Х., Колотилина Н.К., Шайхина С.У. и др. // Сорбиионные и хроматографические проиессы. 2016. Т. 16. № 4. С. 280-293.

7. Долгоносов А.М., Хамизов Р.Х., Колотилина Н.К. // Сорбиионные и хроматографические проиессы. 2018. Т.18. № 6. С. 794-809. https://doi.org/10.17308/sorpchrom.2018.18/60 7

8. Долгоносов А.М., Хамизов Р.Х., Колотилина Н.К. //Журн. аналит. химии. 2019. Т. 74. № $4 . \quad$ C. 285-296. doi: 10.1134/S0044450219030034.

9. Шимановский Н.Л., Епинетов М.А., Мельников М.Я. Молекулярная и нанофармакология. М. Физматлит. 2009. 622 с.

10. Fraum T., Ludwig D., Bashir M., Fowler K. //. J. Magn. Reson. Imaging. 2017. Vol. 46. pp.338-353. https://doi.org/10.1016/jmri. 25625

11. Rogosnitzky M., Branch S. // BioMetals. 2016. Vol. 29. pp. 365-376. doi: 10.1007/s10534-016-9931-7

12. Тоболов А.А, Богатырев В.Л., Журко Ф.В. // Второй всесоюзный симпозиум по термодинамике ионного обмена. Расширенные тезисы докладов. 1975. Минск. С. 50-53. 


\title{
Application of solid-phase ion exchange method for saturation nanoionites with drugs in the absence of a background electrolyte
}

\author{
(C) 2020 Kolotilina N.K., Shaykhina S.U. \\ Institute of Geochemistry and Analytical Chemistry named after V.I. Vernadsky RAS, Moscow
}

The problem of developing systems for targeted drug delivery using nanosized carriers is currently an urgent issue. Therefore, polymeric materials due to their physical properties, high drug transfer capacity, biocompatibility, and low toxicity are of great interest. Nanosized ion exchangers (NIEX) obtained in the form of stable hydrosols (cationic - NIC and anionic - NIA) based on their characteristics have the potential to be used as carriers of ionic forms of drugs.

The aim of this study was the description of the method for obtaining pure dosage forms based on nanoionites by the solid phase ion exchange method, which is possible only if one phase is a macroionite, and the other is a nanoionite of the same polarity.

The method is illustrated by the example of obtaining NIA in the form of an anion of di-N-methylglucamine salt of gadopentate (trade name "Magnevist") - a contrast agent for MRI. The production of targeted drug delivery is important due to the potential toxicity of gadolinium compounds.

An independent study of solid-phase ion exchange for systems with macroionites was carried out. The experiment consisted in the transfer of a certain ion from one anion exchange column (1) in (Br-) form to another, the same, but in OH-form (2), by passing the NIA nanosized hydrosol of known concentration through a system of columns connected in series. The degree of transfer of bromide was controlled by measuring the concentration of $\mathrm{Br}^{-}$washed from the column 2 by sodium alkali solution after separation of the columns. Bromide was determined chromatographically. We determined that the solid-phase ion exchange was complete by balancing the amount of equivalents of the passed NIA and washed bromide. Bromide was completely transferred from column 1 to column 2.

After proving the efficiency of the solid-phase ion exchange method, we applied this approach for the production of particles of NIA nanosized hydrosol loaded with the [Gd-DTPA] ${ }^{2-}$ anion. We used macroanionite and NIA in salt forms, since the gadolinium complex is stable in neutral and weakly acidic media. Control of the completeness of the transfer of AV-17 $\left(\mathrm{Cl}^{-}\right)$into the $[\mathrm{Gd}-\mathrm{DTPA}]^{2-}$ form was carried out by the simultaneous ion chromatographic determination of both anions in the samples taken at the column outlet. Next, the gadolinium complex was transferred to nanosized hydrosol $\left(\mathrm{Br}^{-}\right)$, passing the NIA through the column with macroanionite in the form [Gd-DTPA $]^{2-}$. Thus, in the process of solid-phase ion exchange, the NIA hydrosol was saturated with the $[\mathrm{Gd}-\mathrm{DTPA}]^{2-}$, displacing bromide into the macroanionite phase.

Hydrosol obtained by solid-phase ion exchange in the form of [Gd-DTPA] ${ }^{2-}$ was submitted for testing at the Pirogov Russian National Research Medical University.

Due to the possibilities of solid-phase ion exchange, nanoionites can be easily and quickly converted into the ionic form of a drug, polystyrene nanoparticles can be saturated without losing expensive components in the surrounding solution. The absence of extraneous chemical impurities in the resulting preparation is of primary importance for its medical use, as this minimizes unwanted side effects.

Keywords: solid-phase ion exchange, nanoionites, nanoion exchanger, hydrosol, nanoparticles, targeted drug delivery.

\section{References}

1 Perez-Herrero E., Eu.J Pharm. and Biopharm., 2015, Vol. 93, pp. 52-79. doi: 10.1016/j.ejpb.2015.03.018

2. Duncan R. Vicent M., Adv. Drug Deliv. Rev., 2013, Vol. 65, pp. 60-70. doi: 10.1016/j.addr.2012.08.012

3. Ekladious I., Colson Y, Grinstaff M., Natur. Rev. Drug Discov., 2019, Vol. 18, pp. 273-294. doi: $10.1038 / \mathrm{s} 41573-018-0005-0$
4. Matsumura Y, Maeda H., Cancer Research, 1986, Vol. 46, No 12, pp. 6387-6392.

5. Dolgonosov A.M., Kolotilina N.K., Yadykov M.S., Burmistrov A.A., J. of Analytical Chemistry, 2013, Vol. 68, No 5, pp. 490-496.

6. Dolgonosov A.M., Khamizov R.Kh., Kolotilina N.K., Shaikhina S.U., Evstigneeva P.V., Sorptsionnye i khromatograficheskie protsessy, 2016, Vol. 16, No 4, pp. 280-293. 
7. Dolgonosov A.M., Khamizov R.Kh., Kolotilina N.K.. Sorptsionnye i khromatograficheskie protsessy, 2018, Vol. 18, No 6, pp. 794-809. https://doi.org/10.17308/sorpchrom.2018.18/607.

8. Dolgonosov A.M., Khamizov R.Kh., Kolotilina N.K., J. of Analytical Chemistry, 2019, Vol. 74, No 4, pp. 285-296, doi: 10.1134/S1061934819030031.

9. Shimanovsky N.L., Epinetov M.A., Melnikov M.Ya. Molecular and Nanopharmacology, M.,. Fizmatlit, 2009, 622 p.

Колотилина Надежда Константиновна научный сотрудник, лаборатория сорбционнЫХ методов, ГЕОХИ РАН, Москва

Шайхина Светлана Ураловна - младший научный сотрудник, лаборатория сорбционных методов, ГЕОХИ РАН, Москва
10. Fraum T, Ludwig D., Bashir M., Fowler K., J. Magn. Reson. Imaging., 2017, Vol. 46, pp. 338-353. https://doi.org/10.1016/jmri. 25625

11. Rogosnitzky M., Branch S., BioMetals., 2016, Vol. 29, pp. 365-376. doi: 10.1007/s10534-019-9931-7

12. Tobolov A.A., Bogatyrev V.L., Zhurko F.V., Second All-Union Symposium on Ion Exchange Thermodynamics. Extended abstracts, 1975, Minsk, pp. 50-53.

Kolotilina Nadezhda K. - Scientific associate, Lab of Sorption Methods, GEOKHI RAS, Moscow, e-mail: nkolotilina@mail.ru

Shaykhina Svetlana U. - Scientific associate, Lab of Sorption Methods, GEOKHI RAS, Moscow 